\title{
DETERMINANTS OF FOCUSED ANTENATAL CARE USE AMONG PREGNANT WOMEN, ATTENDING DELIVERY, IN KARAMARA HOSPITAL, JIGJIGA TOWN, SOMALI REGION, EAST ETHIOPIA
}

\author{
HAMDI HIRSI*, MOHAMED MOHAMUD \\ Department of Public Health, College of Medicine and Health Science, Jigjiga University, Jigjiga, Ethiopia. Email: hirsihasan118@gmail.com \\ Received: 24 March 2021, Revised and Accepted: 16 April 2021
}

\section{ABSTRACT}

Objective: The aim of the study was to assess the determinants of focused antenatal care (ANC) use among pregnant women attending delivery in Karamara Hospital at Jigjiga town, Somali region, East Ethiopia from February to March 2017.

Methods: Unmatched case-control study was conduct in Karamara hospital at Jigjiga town. A total of 366 women 183 cases and 183 controls were selected by convenience sampling techniques. Data were collected by trained data collectors using face-to-face interview of women. Logistic regression model was used to determine the predictors of focused ANC nonuse. Variables with $\mathrm{p}<0.05$ and $95 \%$ confidence interval were considered as statistically significant.

Results: The study revealed that $80.3 \%$ of women had ANC follow-up in their last pregnancy. About 39.3\% of women had FANC visit. Urban resident women (AOR=3.1, 95\% CI [1.418, 7.211]), knowledge of danger signs of pregnancy (AOR=3.5, 95\% CI [1.886, 6.832]), knowledge of days FANC services provision (AOR=4.4, 95\% CI [1.86, 10.59]), existence of tradition believe about FANC (AOR=0.16, 95\% CI [0.079, 0.347]), distance less than or equal $1 \mathrm{~h}(\mathrm{AOR}=2.2,95 \% \mathrm{CI}[0.838,5.850])$, existence of health facility (AOR=3.14, 95\% CI [1.38, 7.544]), information form health-care provider (AOR=3.6, $95 \% \mathrm{CI}[1.04,12.83]$ ) delivery than their counterpart, and payment needed to use FANC (AOR=0.138, 95\% CI [0.057, 0.331]) were significantly associated with FANC use.

Conclusion: Being urban resident, knowledge about danger sign of pregnancy, time to reach nearest health facility, radio/Tv use and culture were significantly associated with FANC utilization among pregnant women. Thus, efforts should be made to improve accessibility and availability of the health facility, educating of pregnant women about danger signs of pregnancy physical, information education, and communication of the benefits of FANC service were also critical area that needs intervention.

Keywords: Focused antenatal care, Pregnant women, Determinants, Somali region, Ethiopia.

(C) 2021 The Authors. Published by Innovare Academic Sciences Pvt Ltd. This is an open access article under the CC BY license (http://creativecommons. org/licenses/by/4.0/) DOI: http://dx.doi.org/10.22159/ijms.2021v9i3.41592. Journal homepage: https://innovareacademics.in/journals/index.php/ijms

\section{INTRODUCTION}

\section{Background}

Antenatal care (ANC) is an umbrella term used to describe the medical procedures and care that are carried out during pregnancy the goal-oriented ANC approach was recommended and adopted by the WHO in 2002. Focused ANC approach recognizes two key realities: First, frequent antenatal visits in the old approach do not necessarily improve pregnancy outcomes and second, many women with risk factors may never develop complications. Maternal mortality, the death of women while pregnant or within 42 days of termination of pregnancy, remains disturbing highly in sub Saharan Africa [1].

Focused ANC says that every pregnant woman is at risk for complications, and therefore, all women should get basic care and monitoring for complications. The provision of quality basic care safe, simple cost-effective interventions that all women should receive in turn helps maintain normal pregnancies and can save lives by preventing complications and facilitating early detection and treatment of complications [2].

This model reduces the number of required antenatal visits to four and provides focused services shown to improve maternal outcomes. Focused ANC eliminates the traditional risk assessment and instead emphasizes helping women to maintain normal pregnancies by identifying existing health conditions, detecting emerging complications, promoting health, preparing for a healthy birth, and educating clients on postpartum care including nutrition, breastfeeding, and family planning [3].

In Ethiopia, the maternal mortality was estimated to be 673 deaths per 100,000 live births and infant mortality rate was 77 per 1,000 live births, which is among the highest in the world. As emphasized in the 2011 Ethiopian Demographic Health Survey (EDHS), one explanation for poor health outcomes among women in Ethiopia was non-use of modern health-care services. According to EDHS (2014), FANC service coverage in Ethiopia was very low and little is known about determinants factor of FANC service utilization in Ethiopia and particularly in Somali region where the utilization of maternal health service is $15 \%[4,5]$. Therefore, this study aimed to assess determinants of focused ANC use among pregnant women attending delivery in Karamara Hospital at Jigjiga town, Somali region, East Ethiopia.

\section{METHODS}

\section{Study area and period}

The study was conducted in Jigjiga town, Somali region, East Ethiopia. Jigjiga is the capital city of the Somali region found $576 \mathrm{~km}$ away from the capital city of Ethiopia (Addis Ababa) and there are 22 Kebeles in the town. According to Somali region statistical office, the projected population is 600,$0000 ; 280,000$ were female among this 120,092 were women in reproductive age group and 72,000 deliveries annually and there are one public hospital and two public health centers in the town [6]. The study was conducted in Karamara Hospital from February to March 2017 


\section{Study design}

Unmatched case-control study design was conducted to assess determinants of focused ANC use among pregnant women attending delivery in Karamara Hospital at Jigjiga town, Somali region, East Ethiopia.

\section{Source population}

The source populations were pregnant women who used and did not use focused ANC in their last pregnancy in Jigjiga town public health facilities

\section{Study population}

The study population was pregnant women attending delivery service in Karamara Hospital, Jigjiga town.

\section{Cases}

Pregnant women who have not used focused antennal care for at least 9 months before the start of data collection.

\section{Controls}

Pregnant women used focused ANC in one of the public health facilities and have four or more antennal visits at least 9 months before the start of data collection.

\section{Inclusion and exclusion criteria}

\section{Inclusion criteria}

Pregnant women who were regularly (without being dropouts) using FANC services at least for 9 months before the time of data collection in one public health facilities and pregnant women who were not be using FANC services for at least 9 months before the time of data collections in one of the public health facilities were included in the study.

\section{Exclusion criteria}

Mothers mentally incapable for interview during data collection period were excluded from the study.

\section{Sample size determination}

Sample size was calculated using Epi Info Version 3.5.3 statistical computer program using two population proportions formula based on the following assumption; $95 \%$ confidence level, $80 \%$ power, odds ratio of 1.88 , and case to control ratio of $1: 1$, thus, based on the above findings, it was assumed in this study that, $30.5 \%$ proportion of urban women use FANC [7]. Finally, the calculated sample size was 366 (183 cases and 183 controls) after adding 10\% non-response rate.

\section{Sampling procedure}

Based on the case definition criteria. Using convenience sampling technique, cases and controls were selected from the women who come to the hospital for delivery service until the required sample size obtained.

\section{Data quality management}

Before embarking on data collection, pre-test was conducted in nearest rural Kebeles to ensure the validity of the survey tools and to standardize the questionnaire. Supervisors and the principal investigator were made frequent checks on the data collection.

\section{Data collection procedure}

Data were collected by trained six midwifery and two nurse local data collectors who had previous experience in data collection using face-toface interview administered questionnaire which was developed from reviewing others studies and modified according to context of the study then translated into local language (Af-Somali). Training was given for data collectors and supervisor on collection technique and objectives of the study, questionnaire, sampling methods, and securing informed verbal consent from the study participants for 3 days.

\section{Study variables \\ Dependent variables}

- $\quad$ Focused antenatal care use.

\section{Independent variables}

- Sociodemographic characteristics; age of the women, occupation of women, husband age, husband occupation, husband education, family size, ethnicity, marital status religion, residence

- Knowledge of FANC: Reproductive history and knowledge of obstetric danger signs

- Attitude related; service satisfaction, pregnancy-related traditional barriers, pervious pregnancy history, information about FANC

- Health facility related; health facility around the home, distance of health facility, working days, and health provider related.

\section{Operational definitions}

\section{Focused ANC}

In this study, focused ANC was considered as if a mother received at least four ANC visits and more during pregnancy period by health professional [8].

\section{Knowledge of obstetric danger sign}

Women were considered as good knowledgeable about obstetric danger signs related to pregnancy and childbirth if they scored equal and above the mean (above 3) from five knowledge questions and if less than mean considered as poor knowledgeable [5].

\section{Knowledge of focused ANC}

Refers the level of understanding of mothers' about focused ANC if the mother answers five and above women considered as knowledgeable and below five as poor knowledge.

- Cases; pregnant women who have no FANC

- Controls; pregnant women who have four ANC visits and more

Data analysis procedure

Data entered into EpiData version 3.1 then exported for data analysis using SPSS Version 20.0 statistical software. Descriptive statistics were used to calculate the mean and standard deviation for continuous variables and frequency for categorical variables. Binary logistic regression analysis was used to determine the independent variables effects on dependent variables. Multivariate logistic regressions were performed to assess the effect of independents variables on dependent variables while controlling effect of other factors. Multivariable analysis, statistical significance was considered when $\mathrm{p}=0.05$ and $95 \%$ C.I with adjusted odds ratios to interpret and reported.

\section{Ethical clearance}

Ethical clearance was obtained from the ethnical clearance committees of Institutional Health Research Ethics Review Committee of Haramaya University. An official letter was written to administrative body of Jigjiga City Administration and others concerned bodies of the Kebeles. The objective and importance of the study were explained and informed consent was obtained from each participant. Privacy and confidentiality were maintained at all levels of the study. Participants who are not willing to participate in the study and those who went to quit from the study at any juncture were informed do so without any restrictions.

\section{RESULTS}

A total of 366 respondents were planned to visit and all were successfully interviewed with an overall response rate of $100 \%$.

\section{Sociodemographic characteristics}

The mean age of the cases was 27.33 (S. D \pm 6.315 ) while the mean age of the control was 26.65 (S. D \pm 5.62 ). More than half of the study participants were urban resident in both cases 102 (55.7\%) and controls 127 (69.4\%). By ethnic composition, majority were Somali ethnic group in both cases, case group 160 (87.4) and control 127 (69.4) by religious affiliation, more than two-third of cases 171 (93.4\%) and control $164(89.3 \%)$ were followers of Islam. About two-third of both cases 143 (78.1\%) and controls 140 (80.9\%) were married. Majority of both the cases 112 (61.2) and 113 (61.7) of the controls 
were housewife, majority of husbands in cases 65 (35.5) and 73 (39.3) of the controls had no formal education, 74 (40.4) of cases husband and 80 (43.7) of the controls husband were government workers, while of the 62 (33.4) cases and 76 (41.6) of the controls had family size greater than 7 (Table 1).

\section{Knowledge about FANC}

\section{Knowledge about FANC and danger signs of pregnancy}

Regarding the number of pregnancy, 60 (32.8\%) of the cases and 52 $(28.4 \%)$ of the control were prim gravida, while near half of the cases $79(43.2 \%)$ and controls $85(46.4 \%)$ had $2-4$ pregnancy, the remaining $44(25 \%)$ of the cases and $46(25.1 \%)$ of the controls had more than 4 pregnancies. Two hundred and forty-one $(80.32 \%)$ of the women had ANC follow-up for their last pregnancy, while 70 (39.3\%) of the cases had no ANC follow-up in their last pregnancy; $111(60.7 \%)$ of the cases and 183 (99.9\%) of the controls had ANC follow-up in their last pregnancy. In case of number of FANC visits, 72 (39.3\%) of the case had no any ANC visit, 86 (47\%) of them had one visit, and $25(13.7 \%)$ of the case had two ANC mean while all of the controls had four visit and above. Regarding the number of ANC visits, pregnant women are supposed to have $75(41.6 \%)$ cases and $6(3.3 \%)$ of the controls said

Table 1: Sociodemographic characteristics of pregnant women attending, delivery in Karamara Hospital, Jigjiga town, East Ethiopia, March 2017

\begin{tabular}{|c|c|c|c|c|}
\hline \multirow{2}{*}{$\begin{array}{l}\text { Sociodemographic } \\
\text { variable }\end{array}$} & \multicolumn{2}{|c|}{ Case } & \multicolumn{2}{|c|}{ Control } \\
\hline & Frequency & Percent & Frequency & Percent \\
\hline \multicolumn{5}{|l|}{ Age } \\
\hline $15-19$ & 29 & 15.8 & 24 & 13.1 \\
\hline $20-24$ & 40 & 21.9 & 42 & 23.0 \\
\hline $25-29$ & 39 & 21.3 & 46 & 25.1 \\
\hline $30-34$ & 48 & 26.2 & 50 & 27.3 \\
\hline$>35$ & 27 & 14.8 & 21 & 11.5 \\
\hline \multicolumn{5}{|l|}{ Current residency } \\
\hline Urban & 102 & 55.7 & 127 & 69.4 \\
\hline Rural & 81 & 44.3 & 56 & 30.6 \\
\hline \multicolumn{5}{|l|}{ Ethnicity } \\
\hline Somali & 144 & 78.7 & 144 & 78.7 \\
\hline Oromo & 27 & 14.8 & 26 & 14.2 \\
\hline Amara & 12 & 6.6 & 13 & 7.1 \\
\hline \multicolumn{5}{|l|}{ Marital status } \\
\hline Married & 143 & 78.1 & 148 & 80.9 \\
\hline Widow & 30 & 16.4 & 18 & 9.8 \\
\hline Divorced & 10 & 4.4 & 17 & 9.3 \\
\hline \multicolumn{5}{|l|}{ Religion } \\
\hline Muslim & 171 & 93.4 & 164 & 89.6 \\
\hline Orthodox & 12 & 6.6 & 19 & 10.4 \\
\hline \multicolumn{5}{|l|}{ Educational } \\
\hline Uneducated & 98 & 53.5 & 38 & 20.8 \\
\hline Primary & 51 & 27.8 & 89 & 48.6 \\
\hline Secondary & 24 & 13.1 & 36 & 19.7 \\
\hline College and above & 10 & 5.46 & 20 & 10.9 \\
\hline \multicolumn{5}{|l|}{ Occupation } \\
\hline Housewife & 112 & 61.2 & 113 & 61.7 \\
\hline Gov't worker & 26 & 14.2 & 33 & 18.0 \\
\hline Merchant & 45 & 24.6 & 37 & 20.3 \\
\hline \multicolumn{5}{|l|}{ Husband education } \\
\hline Uneducated & 65 & 35.5 & 73 & 39.3 \\
\hline Primary & 65 & 35.5 & 22 & 12.0 \\
\hline Secondary & 30 & 16.3 & 14 & 7.7 \\
\hline College and above & 25 & 13.6 & 74 & 40.4 \\
\hline \multicolumn{5}{|l|}{ Husband occupation } \\
\hline Gov't worker & 74 & 40.4 & 80 & 43.7 \\
\hline Daily labor & 27 & 14.8 & 22 & 12.0 \\
\hline Farmer & 34 & 18.5 & 44 & 24.1 \\
\hline Merchant & 48 & 26.2 & 37 & 20.2 \\
\hline \multicolumn{5}{|l|}{ Family size } \\
\hline $1-4$ & 50 & 27.3 & 44 & 24.0 \\
\hline $5-6$ & 72 & 39.3 & 63 & 34.4 \\
\hline$\geq 7$ & 62 & 33.4 & 76 & 41.6 \\
\hline
\end{tabular}

that one visit is required, $69(37.7 \%)$ of the cases and $6(3.3 \%)$ of the controls said that two visits are required while 39 (21.3) of the case and 11 (6) of the controls said that three visits are required, majority of the controls 160 (87.4) said that four and above visit are required for the pregnancy. Reason for the FANC use 71 (38.8\%) of the cases and $82(44.8 \%)$ of the controls justified their ANC attendance were due to illness, both $66(36.1 \%)$ of the cases and $63(34.4 \%)$ of the controls said that they were told by their families, $33(18 \%)$ of the case and 28 (15.3) of the controls said due to previous pregnancy problem, 13 $(7.1 \%)$ of the cases and $10(5.5 \%)$ of the controls mentioned due to loss of their previous fetus. Regarding the month started ANC followup, $33(18 \%)$ of the cases and $81(44.3 \%)$ of the controls started ANC on the $3^{\text {rd }}$ month, 81 (44.3\%) of the cases and 90 (49.2) 0 f the controls started ANC at 4-6 months of the pregnancy, $41(22.4 \%)$ of the cases and $8(4.4 \%)$ of the controls started ANC at 7-9 months of pregnancy, and $28(15.3 \%)$ of the cases and $4(2.2 \%)$ of the controls do not know the month started ANC. The role of decision whether to attend ANC or not $69(37.2 \%)$ of the cases and $83(45.4 \%)$ of the controls said only myself, $48(26.2 \%)$ of the cases and $56(30.6 \%)$ of the controls said me and my husband, and $52(28.3 \%)$ of the cases and $59(32.2 \%)$ of the controls said only my husband. In case of pregnancy plan, more than half of the cases 101 (55.2\%) and 125 (68.3\%) of the controls told that their pregnancy was unplanned while $82(44.8 \%)$ of the cases and $57(31.06 \%)$ of the controls said that their pregnancy was unplanned (Table 2).

\section{Danger signs of pregnancy}

Half of the cases 87 (47.5\%) and two-third of the control $126(68.9 \%)$ know persistent vomiting, more than half of the cases 101 (52.5\%) and controls 129 (70.5\%) know vaginal bleeding, 66 (36.1\%) of the cases and $116(63.4 \%)$ of the controls know severe headache, and 65 (35.5\%)

Table 2: Knowledge-related factors affecting the utilization of FANC service among pregnant women attending delivery in Karamara Hospital, Jigjiga town, Somali region, East Ethiopia

\begin{tabular}{lllll}
\hline Variables & Case & Percent & Control & Percent \\
\hline No. of pregnancy & Frequent & Percent & Frequent & Percent \\
1 & 60 & 32.8 & 52 & 28.4 \\
2_4 & 79 & 43.2 & 85 & 46.4 \\
>4 & 44 & 25 & 46 & 25.1 \\
ANC for the last pregnancy & & & \\
Yes & 111 & 60.7 & 183 & 100 \\
No & 70 & 39.3 & 0 & 0 \\
No ANC visits & & & & \\
0 & 72 & 39.3 & 0 & 0 \\
1 & 86 & 47 & 0 & 0 \\
2 & 25 & 13.7 & 0 & 0 \\
4 & & & 183 & 100 \\
1 & 75 & 41.6 & 6 & 3.3 \\
2 & 69 & 37.7 & 6 & 3.3 \\
3 & 39 & 21.3 & 11 & 6 \\
4+ & 0 & 0 & 160 & 87.4 \\
Illness & 71 & 38.8 & 82 & 44.8 \\
Told by family & 66 & 36.1 & 63 & 34.4 \\
Previous problem & 33 & 18 & 28 & 15.3 \\
Fetal loss & 13 & 7.1 & 10 & 5.5 \\
Month started ANC & & & & \\
3 & 33 & 18 & 81 & 44.3 \\
4_6 & 81 & 44.3 & 90 & 49.2 \\
7_9 & 41 & 22.4 & 8 & 4.4 \\
Do not know & 28 & 15.3 & 4 & 2.2 \\
Ask permission & & & & 37.2 \\
Mine only & 83 & 45.4 & 69 & 30.6 \\
Me and my & 48 & 26.2 & 56 & 32.2 \\
husband & & & & 68.3 \\
My husband only & 52 & 28.3 & 59 & \\
Pregnancy plan & & & & \\
Yes & 101 & 55.2 & 125 & \\
No & 44.8 & 57 & \\
\hline & & & & \\
\hline
\end{tabular}


of the cases and 112 (61.2) of the controls know hypertension. Last and $95(51.9 \%)$ of the cases and $139(76.0 \%)$ of the controls know fits and face swelling while $96(52.5 \%)$ of the cases and $57(31.06 \%)$ of the controls do not know persistent vomiting, 82 (44.8\%) of the cases and 54 (29.5\%) of the controls do not know vaginal bleeding, 117 (63.9\%) of the cases and $67(36.6 \%)$ of the controls do not know severe headache, $118(64.5 \%)$ of the cases and $71(38.8 \%)$ of the controls do not know hypertension, and 88 (48.1\%) of the cases and $44(24 \%)$ of the controls do not know fits and face swelling us danger sing of pregnancy (Table 3 ).

\section{Attitude-related factors}

Regarding the source of information, 53 (29\%) case and 71 (38.8\%) of the control got information from health-care provider, $27(14.8 \%)$ of the case and $40(21.9 \%)$ of the control get information from radio, $50(27.3 \%)$ of the case and $27(14.8 \%)$ of the control get information from traditional birth attendants, $40(21.9 \%)$ of the case and 35 $(19.1 \%)$ of the control get information from relatives, and $13(7.1 \%)$ of the case and $10(5.5 \%)$ of the control get information from other sources incase service provided in the health facility; more than half of the case $95(51.9 \%)$ and control 108 (59\%) mentioned the health care worker in the facility were good, $54(29.5 \%)$ of the case and 44 (24\%) of the control said short waiting time, $14(7.7 \%)$ of the case and $13(7.1 \%)$ of the control said HEP were available, and $20(10.9 \%)$ of the cases and $18(9.8 \%)$ of the control said that there is a flexibility of service provision schedules. Regarding the need of payment to use FANC, no any payment charged to use ANC of which 70 (38.3\%) were case and $49(26.8 \%)$ were control, also more than half of participants 250 of which 113 (61.7\%) were case and 137 (73.2\%) were control said that payment is not needed to use FANC. In case of existence of cultural belief about FANC use overall 135 of the study participants, 89 (48.6\%) of case and $46(25.1 \%)$ of control beliefs that there is traditional belief that prevents pregnant women to ANC. While the remaining 94 (51.4\%) of case and 137 (74.9\%) of control reported that there is no traditional belief that prevents pregnant women to use FANC services (Table 4).

\section{Health facility-related factors}

The existence of health facility around their Kebeles was 84 (45.9\%) of the cases and $110(60.1 \%)$ of the controls have health facility in their Kebeles. While 99 (54.1\%) of cases and 73 (39.9\%) of controls have no health facility around their Kebeles. According to the distance of the nearest health facility from their home by walk, $32(17.5 \%)$ of the case and $66(36.1 \%)$ of the control are 30 min away from their home. While $43(23.5 \%)$ of the case and $28(15.3 \%)$ of the control were 45 min away from the nearest health and majority of the case 108 (59\%) and controls $89(48.6 \%)$ were mentioned that the nearest health facility for them were more than $1 \mathrm{~h}$ away. Forty two (23.5\%) of the cases and $38(20.8 \%)$ of the control were reported health facility was far, 39 $(21.5 \%)$ of the case and $46(25.1 \%)$ of the control were reported there

Table 3: Knowledge about danger signs of pregnancy among pregnant women attending delivery in Karamara Hospital, Jigjiga town, East Ethiopia, 2017

\begin{tabular}{lcccc}
\hline Variables & \multicolumn{1}{c}{ Case } & \multicolumn{2}{c}{ Control } \\
\hline $\begin{array}{l}\text { Persistent vomiting } \\
\quad\end{array}$ & 87 & 47.5 & 126 & 68.9 \\
$\quad$ Yes & 96 & 52.5 & 57 & 31.06 \\
$\quad$ No & & & 129 & 70.5 \\
Vaginal bleeding & & 55.2 & 54 & 29.5 \\
$\quad$ Yes & 101 & 44.8 & & \\
$\quad$ No & 82 & & 116 & 63.4 \\
Severe headache & & 36.1 & 67 & 36.6 \\
$\quad$ Yes & 66 & 63.9 & & \\
$\quad$ No & 117 & 35.5 & 112 & 61.2 \\
Hypertension & 65 & 64.5 & 71 & 38.8 \\
$\quad$ Yes & 118 & & & 76 \\
$\quad$ No & 95 & 51.9 & 139 & 24 \\
Face and fit swelling & & 48.1 & 44 & \\
$\quad$ Yes & 88 & & & \\
$\quad$ No & & & & \\
\end{tabular}

is no health care provider in the facility, 65 (35.5\%) of the case and 74 $(40.4 \%)$ of the control were reported lack of medicine in the facility while the remaining $36(19.7 \%)$ of the case and $24(13.1 \%)$ of the control were reported lack of privacy (Table 5). In case of knowledge about the number of days, FANC service is provided in the health facility, majority of case $119(65 \%)$ and controls $150(82 \%)$ reported that ANC service was provided every day, $11(6 \%)$ of the case and $4(2.2 \%)$ of the control reported that ANC service was provided once in week, 18 (9.8\%) of the case and 7 (3.8\%) of the control reported twice in week, while $35(19.1 \%)$ of the case and $22(12 \%)$ of the control reported 3 times in week (Table 4).

\section{Factors associated with FANC service utilization}

The bivariate logistic regression shows urban resident women (COR=3, $95 \%$ CI [1.911, 4.806]), knowledge of danger signs of pregnancy (COR=2.4, 95\% CI $[3.73,1.592])$, knowledge of days FANC services provision (COR=2.3, 95\% CI [1.302, 4.307]), existence of tradition believe about FANC (COR $=0.13,95 \% \mathrm{CI}[0.224,0.75])$, distance $\leq 1 \mathrm{~h}$ (COR=3.5, 95\% CI $[2.178,5.905])$, existence of health facility in the Kebeles (COR $=2.5,95 \%$ CI $[1.659,3.889]$ ), payment needed to use FANC (COR=0.46, 95\% CI [0.285, 0.737]), and information form health-care provider (COR=1.4, 95\% CI [0.611, 3.217]).

Table 4: Attitude-related factors affecting utilization of focused antenatal care among pregnant women attending delivery in Karamara Hospital, Jigjiga town

\begin{tabular}{lcc}
\hline Variables & $\begin{array}{c}\text { Case } \\
\mathbf{n = 1 8 3}(\%)\end{array}$ & $\begin{array}{c}\text { Control } \\
\mathbf{n = 1 8 3}(\%)\end{array}$ \\
\hline Information about ANC & $53(29)$ & $71(38.8)$ \\
Health worker & $27(14.8)$ & $40(21.9)$ \\
Radio & $50(27.3$ & $27(14.8)$ \\
Traditional birth attendants & $40(21.9)$ & $35(19.1)$ \\
$\quad$ Relatives & $13(7.1)$ & $10(5.5)$ \\
Others (specify) & & \\
ANC in the facility & $95(51.9)$ & $108(59)$ \\
$\quad$ Good health worker attitude & $54(29.5)$ & $44(24)$ \\
Short waiting hours & $14(7.7)$ & $13(7.1)$ \\
Availability of staff & $20(10.9)$ & $18(9.8)$ \\
Flexibility of clinic schedules & & \\
ANC payment & $70(38.3)$ & $49(26.8)$ \\
Yes & $113(61.7)$ & $137(73.2)$ \\
No & & \\
Traditional belief of ANC & $89(48.6)$ & $46(25.1)$ \\
Yes & $94(51.4)$ & $137(74.9)$ \\
No & & \\
\hline
\end{tabular}

Table 5: Health facility-related factors affecting the utilization of focused antenatal care in Karamara Hospital, Jigjiga town, East Ethiopia

\begin{tabular}{lcc}
\hline Variable & Cases & Control \\
\hline $\begin{array}{l}\text { Health facility in Kebeles } \\
\text { Yes }\end{array}$ & $84(45.9)$ & $110(60.1)$ \\
No & $99(54.1)$ & $73(39.9)$ \\
Nearest facility & $32(17.5)$ & $66(36.1)$ \\
30 min & $43(23.5)$ & $28(15.3)$ \\
45 min & $108(59)$ & $89(48.6)$ \\
$>1$ h & & \\
Problems of using ANC & $43(23.5)$ & $38(20.8)$ \\
Far of health facility & $39(21.5)$ & $76(25.1)$ \\
Lack of health provider & $65(35.5)$ & $24(13.1)$ \\
Lack of medicine & $36(19.7)$ & $150(82)$ \\
Lack of privacy & $119(65)$ & $4(2.2)$ \\
ANC service offered & $11(6)$ & $7(3.8)$ \\
Every day of the week & $18(9.8)$ & $22(12)$ \\
Once a week & $35(19.1)$ & \\
Twice a week & & \\
Three time a week & &
\end{tabular}


Following the bivariate analysis, multivariate analysis was performed urban resident women (AOR=3.1, 95\% CI [1.418, 7.211]), knowledge of danger signs of pregnancy (AOR=3.5, 95\% CI [1.886, 6.832]), knowledge of days FANC services provision (AOR=4.4, 95\% CI $[1.86$, 10.59]), existence of tradition believe about FANC (AOR=0.16, 95\% CI $[0.079,0.347])$, distance $\leq 1 \mathrm{~h}(\mathrm{AOR}=2.2,95 \%$ CI $[0.838,5.850]$ existence of health facility in the Kebeles (AOR=3.14, 95\% CI [1.308, 7.544]), with payment needed to use FANC (AOR=0.138, 95\% CI [0.057, $0.331]$ ), and information form health-care provider (AOR=3.6, 95\% CI [1.04, 12.83]) (Table 6).

\section{DISCUSSION}

This study was conducted to assess the determinants of focused ANC use among pregnant women attending delivery in Karamara Hospital, Jigjiga town, Somali region, East Ethiopian to effectively tackle the low utilization of focused ANC and its associated problems in Ethiopia in general and in the present study areas in particular, there is a need to investigate the contribution of a number of factors influencing the utilization of focused ANC. Accordingly, this study has attempted to look into differentials of focused ANC use among users and none users by the World Health Organization; the users of focused ANC are those who have four visits and above while the none users are those who have less than counterparts.

In this study, the residency of the participant was one of the factors showed association with the use of FANC services, urban women were 3.1 times more likely to use FANC service than the rural women $\{\mathrm{AOR}=3.1,95 \%[1.418,7.211]\}$ this is consistence with Jalal et al. [9]. This might be pregnant women may find it difficult to travel in rural areas, especially when the condition of the roads is poor. Shortages of skilled attendants are common throughout developing countries, especially in rural areas. In general, there is a lack of adequate staff in rural areas compared with cities.

Another predictor of focused ANC use was knowledge of month/ weeks on starting ANC. Mothers who know the appropriate time for initiating ANC visits were 4.4 times more likely to follow FANC than mothers who did not know the appropriate time for starting ANC visits $(\mathrm{AOR}=4.4,95 \% \mathrm{CI}[1.86,10.59])$. This finding was in line with a study conducted in Debre Berhan [10-12]. This might be due to when mothers started ANC visit very soon the probability of reaching four and above ANC will be high but if mother started late, they will get delivery before reaching four visits and if their first ANC visit early and providers inform and advise the pregnancy danger signs and services, she will receive that also enhance mothers to get FANC service.

The result of this study showed that knowledge of danger signs during pregnancy was significantly associated with focused ANC use [AOR=3.5, 95\% CI $(1.886,6.832)]$. This finding was consistent with a study conducted in Yem special Wereda, Southwest Ethiopia [12,13]. It may be due to mothers who had knowledge on danger signs there may be aware of the possible adverse event happened during pregnancy period that leads mothers follow to FANC than mothers with poor knowledge of danger signs.

Time to reach the nearest health facility significantly determine FANC utilization mothers who traveled $\leq 1 \mathrm{~h}$ from their home to health center were 2 times more likely to follow focused ante natal

Table 6: Factors associated with FANC use among pregnant women attending delivery ward, karamara Hospital Jigjiga town, Somali region, eastern Ethiopia 2017

\begin{tabular}{|c|c|c|c|c|c|}
\hline Variables & Case (without) N (\%) & Control (with) N (\%) & Crude OR (95\% CI) & P-Value & Adjusted OR (95\% CI) \\
\hline \multicolumn{6}{|l|}{ Information sources } \\
\hline Health worker & $53(29)$ & $71(38.8)$ & 1 & 0.426 & 1 \\
\hline Radio & $27(14.8)$ & $40(21.9)$ & $1.402(0.611,3.217)$ & 0.453 & $3.6(1.04,12.83)^{*}$ \\
\hline TraditionalBirth Attendants & $50(27.3)$ & $27(14.8)$ & $1.410(0.575,3.460)$ & 0.016 & $2.3(0.643,8.310)^{*}$ \\
\hline Relatives & 40 (21.9) & $35(19.1)$ & $0.316(0.124,0.804)$ & 0.468 & $0.97(0.240,3.92)$ \\
\hline Others (Specify) & $13(7.1)$ & $10(5.5)$ & $0.720(0.296,1.750)$ & 0.847 & $0.985(0.277,3.525)$ \\
\hline \multicolumn{6}{|l|}{ Payment to use FANC } \\
\hline No & $113(61.7)$ & $137(73.2)$ & $0.46(0.295,0.737)$ & & 1 \\
\hline \multicolumn{6}{|l|}{ Traditional belief of FANC } \\
\hline Yes & $89(48.6)$ & $46(25.1)$ & 1 & 0.001 & $0.16(0.079,0.347)^{*}$ \\
\hline No & $94(51.4)$ & 137 (74.) & $0.13(0.224,0.75)$ & & 1 \\
\hline \multicolumn{6}{|l|}{ Health facility in Kebeles } \\
\hline Yes & $84(45.9)$ & $110(60.1)$ & 1 & 0.001 & $3.141(1.308,7.544)$ \\
\hline No & $99(54.1)$ & $73(39.9)$ & $2.5(1.659,3.889)$ & & 1 \\
\hline \multicolumn{6}{|l|}{ Travelling time } \\
\hline$>45 \mathrm{~min}$ & $43(23.5)$ & $28(15.3)$ & $3.5(2.178,5.905)$ & & $0.237(0.074,0.766)$ \\
\hline$>1 \mathrm{~h}$ & $108(59)$ & $89(48.6)$ & $0.56(0.304,1.052)$ & & 1 \\
\hline \multicolumn{6}{|l|}{ Problems of using F ANC } \\
\hline Far of health facility & $43(23.5)$ & $38(20.8)$ & 1 & 0.029 & 1 \\
\hline Lack of health provider & 39 (21.5) & $46(25.1)$ & $1.5(0.733,3.06)$ & 0.268 & $5.8(1.811,19.18)^{*}$ \\
\hline Lack of medicine & $65(35.5)$ & $74(40.4)$ & $2.4(1.211,4.87)$ & 0.012 & $3.16(1.093,9.381)^{*}$ \\
\hline Lack of privacy & $36(19.7)$ & $24(13.1)$ & $2.32(1.223,4.44)$ & 0.010 & \\
\hline \multicolumn{6}{|l|}{ Days FANC service offered } \\
\hline Every day of the week & $119(65)$ & $150(82)$ & 1 & 0.0001 & $4.44(1.86,10.59)^{*}$ \\
\hline Twice a week & $18(9.8)$ & $7(3.8)$ & $2.3(1.302,4.307)$ & 0.005 & $2.1(0.297,14.95)$ \\
\hline Three time a week & $35(19.1)$ & $22(12)$ & $0.194(0.926,0.41)$ & 0.999 & 1 \\
\hline \multicolumn{6}{|l|}{ Current residency } \\
\hline Urban & $102(55.7)$ & $127(69.4)$ & $3(1.911,4.806)$ & 0.0001 & $3.1(1.418,7.211)^{*}$ \\
\hline Rural & $81(44.3)$ & $56(30.6)$ & 1 & & 1 \\
\hline \multicolumn{6}{|l|}{ Knowledge about danger sings } \\
\hline Knowledgeable & $87(47.5)$ & $96(53.5)$ & $2.4(1.592,3.73)$ & 0.0001 & $3.5(1.886,6.832)^{*}$ \\
\hline Not knowledgeable & $126(68.8)$ & $57(22.2)$ & 1 & & 1 \\
\hline
\end{tabular}

$\mathrm{NB}^{*}=\mathrm{p}<0.05$ 
care than those who travelled more than $1 \mathrm{~h}$ to reach the nearest health institution COR $=2.2,95 \%$ CI $(5.850,0.838)$. The findings are consistent with other study in Kham district, women who lived nearby were 2.9 times more likely to frequent visit ANC than those who lived far away [14-16]. Longer time to reach the nearest health facility discourages mothers to follow FANC because of when they reach 3 and 4 trimesters they are weak to travel long distance in the case of rural area where no transport access in contrary physical proximity of health institution was determining factor in case of this study where most of study participants $96.5 \%$ was travel on foot. Mothers living in closer proximity to modern health services were more likely to receive services from health personnel for the treatment of lifethreatening and high-risk conditions and frequent ANC visit during the pregnancy period.

The result shows that women who had health facility around their home were 3.14 more likely to use FANC services than those do not have (AOR=3.14, 95\% CI $[7.544,1.308])$. This finding is similar the following studies $[8,13,17]$.

Existence of cultural belief about FANC was significantly associated with the use of FANC service in this study women who belief in culture were $84 \%$ less likely to use FANC service than those who do not belief culture $(\mathrm{COR}=0.16,95 \%$ CI $[0.079,0.347])$. This finding was similar with Ugalahi et al. JHIPEGO [6,9]. The possible explanation may because of the perception that the modern health-care sector is intended for curative services only.

Study participants who beliefs payment is needed negatively associated with the FANC utilization [AOR=0.138, 95\% CI $(0.057-0.331)]$. This finding was consistence $[18,19]$, the costs of the service including transportation and necessary laboratory tests may be major factors prohibiting service utilization.

The study revealed that source of information was significantly associated with the utilization of focused ANC, those who received information form radio/Tv were 2.3 more likely to use FANC than others (AOR=2.3, 95\% CI $[0.643,8.310])$, this is similar with studies conducted in Nepal and India [16]. This may be women who watch or listen radio have more information than those do not listen.

\section{Limitation of this study}

Women might experience recall bias, particularly on the services, they had got during their previous obstetrics such as during ANC visit. The study relied on self-report in the utilization of FANC services. Furthermore, it can't establish cause and effect relationships.

\section{CONCLUSION AND RECOMMENDATION}

\section{Conclusion}

Urban resident women, knowledge about danger signs of pregnancy, time to reach the nearest health facility, radio or TV, having health facility in their Kebeles, culture, and need of service charge were significantly associated with the utilization of FANC among pregnant women.

\section{Recommendation}

- Jigjiga city administration should have to improve the availability and accessibility of health facilitates to avert low utilization of FANC service in Jigjiga town rural area

- Mother should educated on the danger signs of pregnancy and initiating time of focused ANC

- Regional health bureau should have to expand and strength health infrastructure to the community nearby this may reduce time to reach health institution and encourage pregnant women to fully utilize FANC services

- IECs approach awareness raising should be implemented to remove cultural beliefs affecting the utilization of FANC services at community level.

\section{ACKNOWLEDGMENT}

We would like to acknowledge all my colleagues and experts from different institutions that helped me in providing valuable information and supports to undertake this study.

\section{AUTHORS' CONTRIBUTIONS}

Hamdi Hirsi and MM were designed; analyzed data, interpreted findings, and drafted the manuscripts and submitted to the journal.

\section{ETHICAL DISCLOSURE}

The protocol of the study was reviewed and approved by College of Medicine and Health Sciences, Jigjiga University. Informed consent was obtained from each participant after explaining the purpose of the study and before starting the interview.

\section{CONFLICTS OF INTEREST}

No competing interest.

\section{REFERENCES}

1. Abosse Z, Woldie M, Ololo S. Factors influencing antenatal care service utilization in Hadiya Zone. Ethiop J Health Sci 2010;20:75-82.

2. Agus Y, Horiuchi S. Factors influencing the use of antenatal care inrural West Sumatra, Indonesia Agus and Horiuchi. BMC Pregnancy and Childbirth 2010;12:1-8

3. Berhe KK, Welearegay HG, Abera GB, Kahsay HB, Kahsay AB. Assessment of antenatal care utilization and its associated factors among 15 to 49 years of age women in Ayder KebelleMekelle city. Am J Adv Drug Deliv 2014;2:62-75.

4. Central Statistical Authority. Ethiopia Demographic and Health Survey 2011. Addis Ababa, Ethiopia: Central Statistical Authority; 2011.

5. Central Statistical Authority. Ethiopia Demographic and Health Survey 2014. Addis Ababa, Ethiopia: Central Statistical Authority; 2014.

6. Ugalahi LO, Yusuf OB, Akinyemi JO, Adebowale AS. Regional differences in the optimal utilisation of antenatal care in Nigeria. Sci J Public Health 2016;4:43-8.

7. Zegeye AM, Bitew BD, Koye DN. Prevalence and determinants of early antenatal care visit among pregnant women attending antenatal care in Debre Berhan Health Institutions, Central Ethiopia. Afr J Reprod Health 2013;17:130-6.

8. World Health Organization. Adolescent Health and Development in the Africa Region. Brazzaville: World Health Organization; 2003.

9. Jalal S, Shah NA. Ante Natal Care (ANC) seeking behavior among women living in an urban squatter settlement: Results from an ethnographic study. Ital J Public Health 2011;8:261-7.

10. Gedefaw M, Muche B, Aychiluhem M. Current status of antenatal care utilization in the context of data conflict: The case of Dembecha district, Northwest Ethiopia. Open J Epidemiol 2014;4:208-16.

11. Ministry of Health. Federal Democratic Republic of Ethiopia. Quarterly Health Bulletin. New Delhi: Ministry of Health; 2014.

12. JHIPEGO. Birth Preparedness and Complication Readiness. A Matrix of Shared Responsibilities. Baltimore: JHIPEGO; 2001.

13. Tewodros B, Mariam AG, Dibaba Y. Factors affecting antenatal care utilization in yem special woreda, Southwestern Ethiopia. Ethiop J Health Sci 2009;19:45-51.

14. Ransom E, Yinger N. Making Motherhood Safer: Overcoming Obstacles on the Pathway to Care. Washington, DC: Population Reference Bureau; 2002.

15. Tariku A, Melkamu Y, Kebede Z. Previous utilization of service does not improve timely booking in antenatal care: Cross sectional study on timing of antenatal care booking at public health facilities in Addis Ababa. Ethiop J Health Sci 2010;24:226-33.

16. Ye Y, Yoshida Y, Harun-Or-Rashid M, Sakamoto J. Factors affecting the utilization of antenatal care services among women in Kham District, Xiengkhouang Province, Lao PDR. Nagoya J Med Sci 2010;72:23-33.

17. USAID. Focused Antenatal Care: Providing Integrated, Individualized Care during Pregnancy; 2007. Available from: http://www. accesstohealth.org/toolres/pdfs/accesstechbrief_fanc.pdf.

18. World Health Organization. Mother-baby Package: Implementing Safe Motherhood in Countries. Geneva: World Health Organization; 1996.

19. World Health Organization. Mother-baby Package: Implementing Safe Motherhood in Countries. Geneva: World Health Organization; 2002. 